Check for updates

Cite this: RSC Adv., 2019, 9, 1792

Received 29th October 2018

Accepted 2nd January 2019

DOI: $10.1039 / \mathrm{c} 8 \mathrm{ra0} 8935 \mathrm{c}$

rsc.li/rsc-advances

\section{Effect of external electric field on nanobubbles at the surface of hydrophobic particles during air flotation}

\author{
Leichao Wu, Yong Han, (D)* Qianrui Zhang and Shuai Zhao
}

In this paper, the effect of external electric field on nanobubbles adsorbed on the surface of hydrophobic particles during air flotation was studied by molecular dynamics simulations. The gas density distribution, diffusion coefficient, viscosity, and the change of the angle and number distribution of hydrogen bonds in the system with different amounts of gas molecules were calculated and compared with the results without an external electric field. The results show that the external electric field can make the size of the bubbles smaller. The diffusion coefficient of the gas increases and the viscosity of the system decreases when the external electric field is applied, which contribute to the reduction of the size of the nanobubbles. At the same time, comparing with the results under no external electric field, the angle of hydrogen bonding under the external electric field will increase, and the proportion of water molecules containing more hydrogen bonds will reduce, which further explains the reason why the external electric field reduces the viscosity. The conclusions of this paper demonstrate at the micro level that the external electric field can enhance the efficiency of air-floating technology for the separation of hydrophobic particles, which may provide meaningful theoretical guidance for the application and optimization of electric field-enhanced air-floating technology in practice.

\section{Introduction}

Since Parker ${ }^{1}$ first observed the presence of nanobubbles using atomic force microscopy (AFM) 20 years ago, nanobubbles formed on the liquid-solid surface quickly attracted widespread attention..$^{2-5}$ Nanobubbles have many unique properties, such as long lifetimes, slow dissolution rates and large specific surface areas etc. ${ }^{6-8}$ Many hypotheses have been proposed to explain these properties, for instance, line tension theory, ${ }^{9}$ dynamic equilibrium model theory, ${ }^{10}$ impurity layer theory, ${ }^{11}$ three-phase contact line theory. ${ }^{12}$ But there is no theory that can perfectly explain the problems of nanobubbles.

Although the mystery of nanobubbles at the theoretical level still needs further research, the separation of hydrophobic solid particles by bubbles in mineral flotation has been widely applied. $\mathrm{TaO}^{13,14}$ found the use of hydrodynamic cavitation to form bubbles on coal surfaces in recent flotation research, which can significantly improve the efficiency of flotation recovery. Hampton ${ }^{15}$ pointed out that the effect of highconcentration salt environment on the bubbles formed by mineral flotation is negligible, but the surface characteristics of solid particles have undergone tremendous changes. Fan ${ }^{16}$ found that nanobubbles with smaller diameters can

Measurement Technology and Instrumentation Key Laboratory of Hebei Province, School of Electrical Engineering, Yanshan University, Qinhuangdao 066004, P. R. China.E-mail: hanyong@ysu.edu.cn significantly reduce the rate of bubble rise and create more favorable conditions for the flotation of hydrophobic particles. It can be seen that the size of the nanobubble and the environment of the solution will have an impact on the flotation effect. Therefore, how to improve the separation efficiency of hydrophobic particles by bubbles in mineral flotation has become the focus of attention.

In recent years, with the development of physical water treatment technology ${ }^{17-19}$, the combination of an external electric or magnetic field and flotation technology has provided a new field. Thiebaut ${ }^{20}$ found that the electromagnetic field enhances the effect of the collector on the flotation of fluorite ore. Birinci ${ }^{21}$ studied the flotation of a binary mixture of quartz and magnetite under the action of a magnetic field. The results shown that the magnetic field can significantly improve the separation efficiency. Murugananthan ${ }^{22}$ studied the method of separating suspended solids, sulfides and other contaminants by electric field flotation technology. The study found that the method is at least $20 \%$ more efficient than the traditional method. Although experiments have shown that an external electric or magnetic field can improve the efficiency of flotation in mineral separation, its mechanism of action remains unclear.

Therefore, the effects of external electric field on nanobubbles adsorbed on the surface of hydrophobic particles were studied by molecular dynamics simulation. The mechanism of the external electric field on the efficiency of air-floating 
separation was explored from the micro level, which may provide a useful guidance for combination of electric field and air-floating technology.

\section{Model and simulation details}

In order to mimic the adsorption of nanobubbles on hydrophobic particles, according to existing research methods, ${ }^{23,24}$ a box of $5.82 \mathrm{~nm} \times 5.88 \mathrm{~nm} \times 5.74 \mathrm{~nm}$ which is first filled with 6010 water molecules was built. The SPCE $^{25}$ model was used for water molecules, because it has been proved to have good performance in the study of nanobubbles at hydrophobic solidwater interface. ${ }^{26}$ Then, the graphene model ${ }^{27,28}$ was used to replace the surface of hydrophobic particles on the bottom of the box, this method have been widely used in the molecular dynamic simulation. ${ }^{23,24}$ Finally, the 60, 120 and $180 \mathrm{CO}_{2}$ molecules were randomly placed to form a gas-liquid-solid three-phase system with different amounts of gas molecules. The EPM2 carbon dioxide potential energy model ${ }^{29}$ was adopted as gas molecule, because it provides good prediction of the different situation ${ }^{30,31}$ (in a wide range of temperatures and pressures). Fig. 1 is a schematic diagram of the initial model.

The OPLS-AA force field ${ }^{32}$ was adopted in all simulations. The force field parameters of graphene, water and gas molecule used in this study are summarized in Table 1.

All the simulations were performed with the GROMACS package (version 5.1.2). ${ }^{33}$ The time step for leapfrog method was $1 \mathrm{fs}$, the system temperature was controlled at $300 \mathrm{~K}$, using velocity rescaling. Particle mesh Ewald $(\mathrm{PME})^{34}$ was used to calculate the long-range electrostatic interaction. The cut-off distance for Lennard-Jones interactions was $1.2 \mathrm{~nm}$. To avoid the unpredictable effect of high electric field intensity on water molecules and graphene. ${ }^{35,36}$ The electric field intensity of $0.1 \mathrm{~V} \mathrm{~nm}^{-1}$ was applied along a given direction (corresponding to the $y$-axis in Fig. 1, parallel to the graphene membrane). All

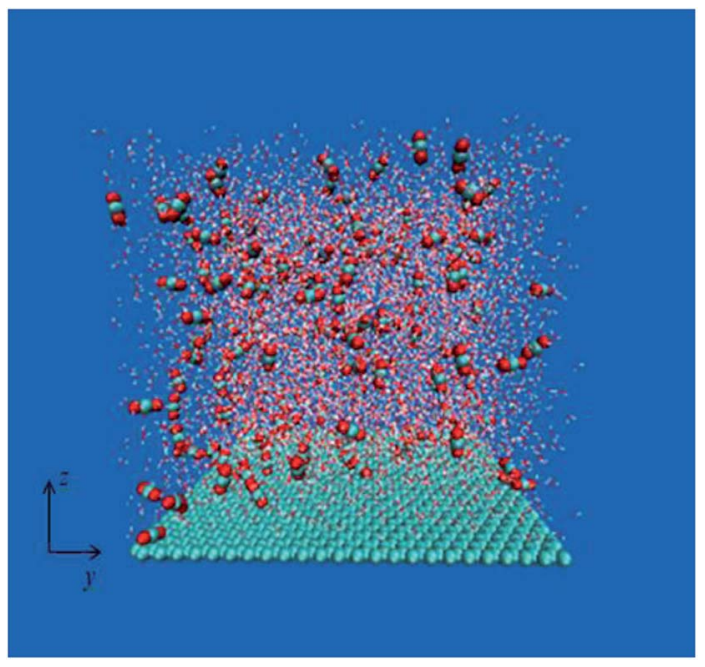

Fig. 1 Schematic diagram of the initial configuration of the simulation. For clarity, the red ball represents the $\mathrm{O}$ atom, the cyan ball represents the $\mathrm{C}$ atom, and the water molecule is given in the form of a line.
Table 1 Parameters used in the simulations

\begin{tabular}{lllll}
\hline Atom & Molecule & $\sigma(\mathrm{nm})$ & $\varepsilon\left(\mathrm{kJ} \mathrm{mol}^{-1}\right)$ & charge $(e)$ \\
\hline $\mathrm{O}$ & Water & 0.3166 & 0.6502 & -0.8476 \\
$\mathrm{H}$ & Water & 0 & 0 & 0.4238 \\
$\mathrm{C}$ & Graphite & 0.3400 & 0.3700 & 0 \\
$\mathrm{CO}$ & Carbon dioxide & 0.2757 & 0.2338 & 0.6512 \\
OC & Carbon dioxide & 0.3033 & 0.6690 & -0.3256
\end{tabular}

the simulations including $500 \mathrm{ps}$ for equilibrium period and 10 ns for sampling stage was used to calculate the properties.

\section{Results and discussion}

\subsection{Density distribution of gas}

The adsorption of bubbles on the hydrophobic particles produces different shapes. ${ }^{37-40}$ Different shapes affect the size of the contact area between bubbles and the hydrophobic particles and the contact time. Under the action of the external electric field, the shape of the bubble will also change. ${ }^{41}$ Therefore, it is necessary to explore the shape change of the bubble under the external electric field. In this paper, the morphology of bubbles on the hydrophobic interface in a solution system with different $\mathrm{CO}_{2}$ dissolved gases under the external electric field were studied. The simulation results are shown in Fig. 2.

It can be seen from Fig. 2 that different numbers of $\mathrm{CO}_{2}$ gas molecules form cylindrical bubbles. In order to study the change of bubble shape in the case of different dissolved gas volume without external electric field, the upper surface of graphene is defined as $0 \mathrm{~nm}$, and the $\mathrm{CO}_{2}$ density profile along the $z$ axis constructed by dividing the simulation box into small boxes with a desired thickness $(0.05 \mathrm{~nm})$ along the $z$ axis. The calculated molecular density profile of $\mathrm{CO}_{2}$ gas is shown in Fig. 3. "60M", "120M", "180M" represent system models containing 60, 120 and $180 \mathrm{CO}_{2}$ molecules, respectively.

In Fig. 3, the abscissa represents the distance perpendicular to the graphene membrane (along the $z$-axis direction), the ordinate represents number density of $\mathrm{CO}_{2}$. Each peak of the curves indicates that in the relevant small box (with a desired thickness of $0.05 \mathrm{~nm}$ ) the $\mathrm{CO}_{2}$ is present. The results show that the density profile of the gas consists of several peaks, which is consistent with the results of Hong's research. ${ }^{26}$ According to Fig. 3 , as the gas content in the system increases, the height of the bubble with the hydrophobic substrate (the position at which the last peak is located) also increases.

The change of the bubble shape caused by the external electric field is reflected in the peak of the density profile. The numerical changes in the peak positions of the system containing the dissolved amounts of different gases are listed in Table 2.

The results in Table 2 show that the peak value of $\mathrm{CO}_{2}$ density profile under an external electric field is smaller than that without the electric field, and as the position away from the hydrophobic substrate is further, both peaks tend to be equal. In all systems in which $\mathrm{CO}_{2}$ molecules are dissolved, the decrease of the peak value means that the bubble size is smaller, 

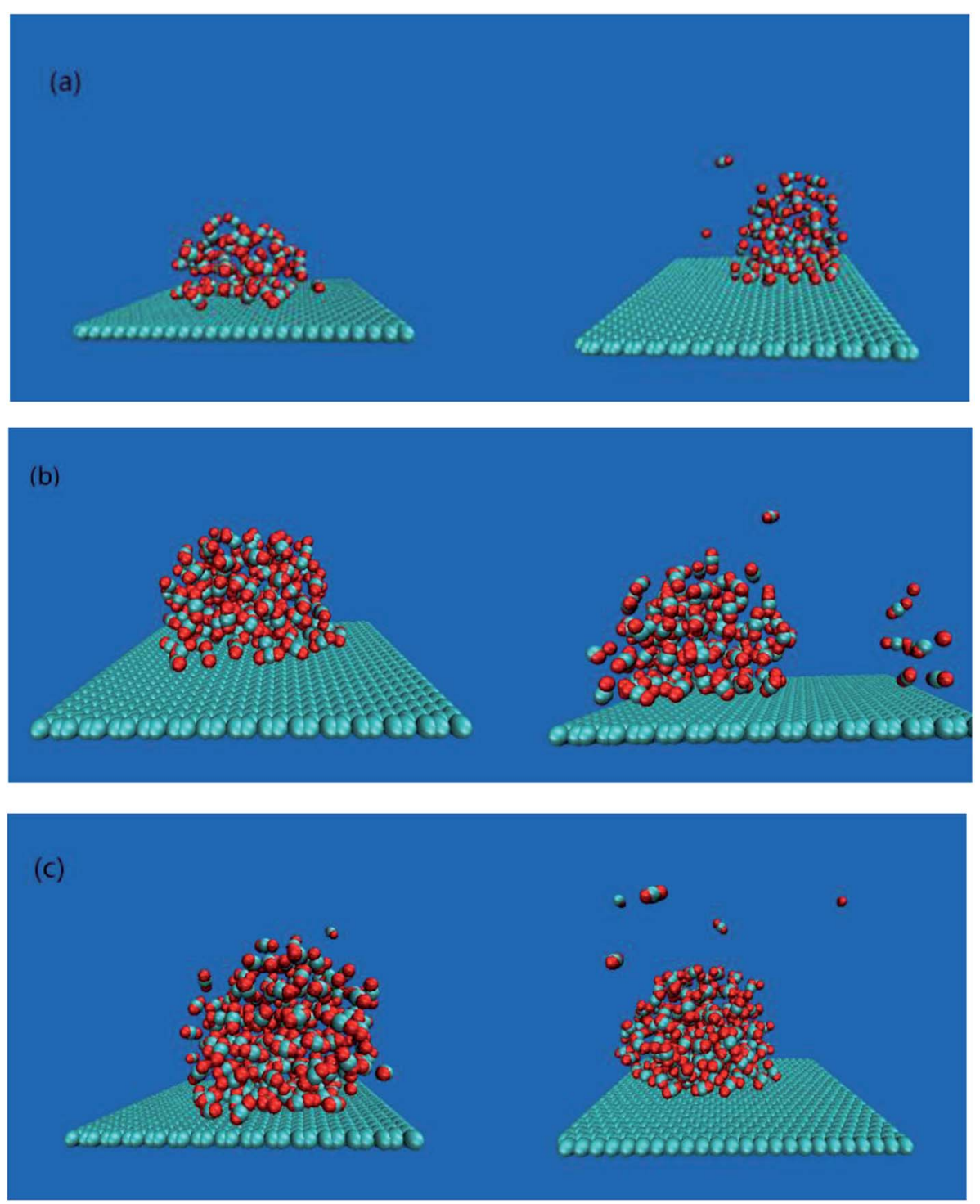

Fig. 2 Snapshot of bubbles under different systems. (a), (b) and (c) are bubbles formed by a system containing 60,120 and $180 \mathrm{CO}_{2}$ molecules respectively, the left side is a bubble without an external electric field, and the right side is a bubble under an external electric field.

the gas content inside the bubble becomes less, and a small amount of gas diffuses into the aqueous phase (as shown in Fig. 2(a)-(c)). Therefore, the results show that the size of bubbles is smaller when the electric field is applied in the process of air flotation, which promotes the combination of bubbles and hydrophobic particles and is beneficial to the adsorption and separation of hydrophobic particles by bubbles.

\subsection{Self-diffusion coefficients}

The diffusion coefficient is an important parameter of the gas in the aqueous solution, and it is a physical quantity that characterizes the gas diffusion ability. In this paper, the diffusion coefficient of $\mathrm{CO}_{2}$ is calculated using the "Einstein" relation: ${ }^{\mathbf{4 2}}$

$$
D=\lim _{t \rightarrow \infty} \frac{1}{6 t}\left[r_{i}(t)-r_{i}(0)\right]^{2}
$$

where $r_{i}(t)$ is the molecule position $i$ at time $t$, hence $r_{i}(0)$ is the initial position and the bracket represents the ensemble average.

Fig. 4 shows the self-diffusion coefficient of $\mathrm{CO}_{2}$ in systems with different amounts of gas dissolved under the external electric field and without an external electric field. It is known from Fig. 4 that the self-diffusion coefficient of $\mathrm{CO}_{2}$ becomes smaller and smaller with the increase of the dissolved amount of gas in the system regardless of whether or not an external electric field exists. When the amount of gas dissolved in the system is constant, the external electric field increases the selfdiffusion coefficient of $\mathrm{CO}_{2}$ compared to that without an electric field. It is found from Fig. 3 that the contact radius of the bubble increases as the amount of dissolved gas increases. According to the Yang-Laplace equation, ${ }^{5}$ the larger the radius of the bubble leads to the smaller the internal pressure. Therefore, we conclude that the decrease of the internal pressure of the 


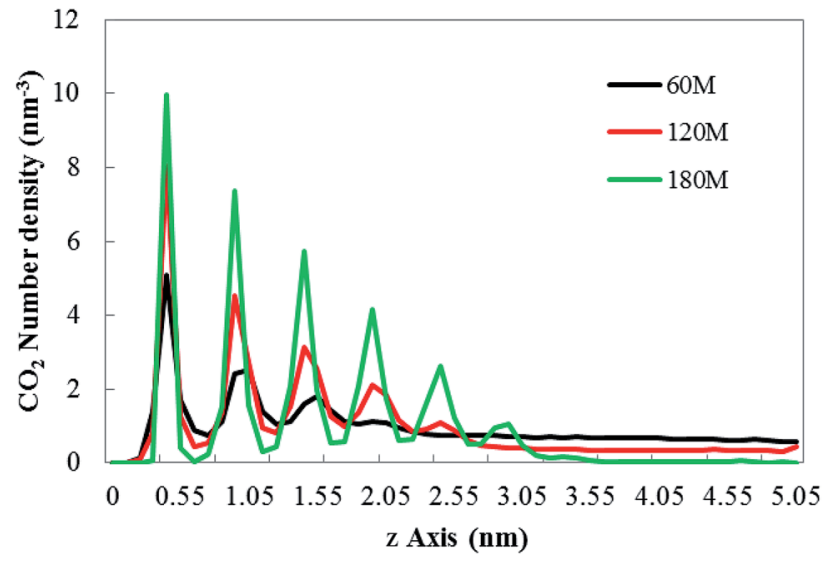

Fig. 3 The $\mathrm{CO}_{2}$ density profile along the $z$ axis under no external electric field. "60M", "120M", "180M" represent system models containing 60,120 and $180 \mathrm{CO}_{2}$ molecules in this paper, respectively.

bubble is the main reason for the decrease of the self-diffusion coefficient of $\mathrm{CO}_{2}$ as the amount of dissolved gas increases. To investigate this phenomenon, we calculated the geometrical parameters (the inside pressure and the volume ${ }^{43}$ of nanobubbles. It can be obtained from following equations:

$$
R_{\mathrm{c}}=\frac{\left(R_{\mathrm{b}}\right)^{2}+H^{2}}{2 H}
$$

where $R_{\mathrm{c}}$ is the radius of curvature, $H$ is the height, $R_{\mathrm{b}}$ is the base radius.

$$
P_{i}=P_{\mathrm{o}}+\Delta P=P_{\mathrm{o}}+\frac{2 r}{R_{\mathrm{c}}}
$$

where $P_{\mathrm{i}}$ is the inside pressure, $P_{\mathrm{o}}$ is the outside pressure, $\Delta P$ is the pressure difference between $P_{\mathrm{i}}$ and $P_{\mathrm{o}}, r=0.072 \mathrm{~N} \mathrm{~m}^{-1}$ is the surface tension of the water/gas interface. ${ }^{43}$

$$
V_{\mathrm{i}}=\pi \times H \times \frac{3\left(R_{\mathrm{b}}\right)^{2}+H^{2}}{R_{\mathrm{c}}}
$$

where $V_{\mathrm{i}}$ is the volume of the bubbles.

The results are listed in Table 3

According to Table 3, we found that the bubble volume in the system increases as the amount of gas increases whether there is an external electric field or not. This result can meet with our conclusion that the increase of the amount of gas leads to the decrease of internal pressure of bubbles.
Under the external electric field, Fig. 4 shows that the diffusion coefficient of the gas becomes larger, so the fluidity of the gas inside the bubble becomes larger than when there is no external electric field. Therefore, we conclude that the increase of gas flow is the main reason for the bubble size becoming smaller. According to the dynamic equilibrium mechanism proposed by Lohse: ${ }^{24,44}$ the outflow of gas in the nanobubble and the inflow of gas will reach a dynamic equilibrium, and the outflow of the gas is proportional to the diffusion coefficient of the gas in the water. After reaching equilibrium, there will be a stable contact radius. During the air-floating process, the gas in the bubble is more active under the external electric field, the outflow of the gas in the bubble becomes larger, the contact radius of the bubble becomes smaller. The smaller bubble is more easily adsorbed on the hydrophobic particle.

\subsection{Viscosity}

Viscosity is a kinetic property that can affect the diffusion rate and conformational change of a molecule in an aqueous solution. The external electric field has an effect on the viscosity of the mixed system..$^{45}$

In this investigation, we used periodic perturbation method $^{46}$ to calculate the viscosity of the system for nonequilibrium dynamic simulation. The Nose-Hoover extended ensemble for temperature coupling and the Parrinello-Rahman method for pressure coupling were used for calculating the viscosity. The viscosity $(\eta)$ can be calculated with the aid of the following equation:

$$
\eta=\frac{A}{V} \frac{\rho}{k^{2}}
$$

where $\rho$ and $V$ are the density and velocity, respectively; $A$ is a constant ${ }^{47}$ and we know the cosine satisfies both the conditions:

$$
a_{x}=A \cos (k z), \quad k=\frac{2 \pi}{l_{z}}
$$

where $l_{z}$ is the height of the box. The instantaneous $V$ in the

\begin{tabular}{|c|c|c|c|c|c|c|}
\hline \multirow[t]{2}{*}{ Without electric field } & $60 \mathrm{M}$ & 5.09 & 2.52 & 1.82 & 1.10 & 0.76 \\
\hline & $120 \mathrm{M}$ & 8.00 & 4.54 & 2.82 & 1.75 & 0.77 \\
\hline \multirow[t]{3}{*}{ Under electric field } & $60 \mathrm{M}$ & 5.00 & 2.39 & 1.80 & 1.10 & 0.76 \\
\hline & $120 \mathrm{M}$ & 7.89 & 4.35 & 2.80 & 1.76 & 0.78 \\
\hline & $180 \mathrm{M}$ & 9.67 & 6.98 & 5.37 & 4.03 & 2.61 \\
\hline
\end{tabular}
simulation is defined as a Fourier coefficient:

$$
V(t)=\frac{2 \sum_{i=1}^{N} m_{i} v_{i, x}(t) \cos \left[k r_{i, z}(t)\right]}{\sum_{i=1}^{N} m_{i}}
$$

Table 2 The value of each peak of the $\mathrm{CO}_{2}$ density profile in different systems ${ }^{a}$

$a$ "Position" represents the abscissa of each peak in $\mathrm{CO}_{2}$ density profile. 


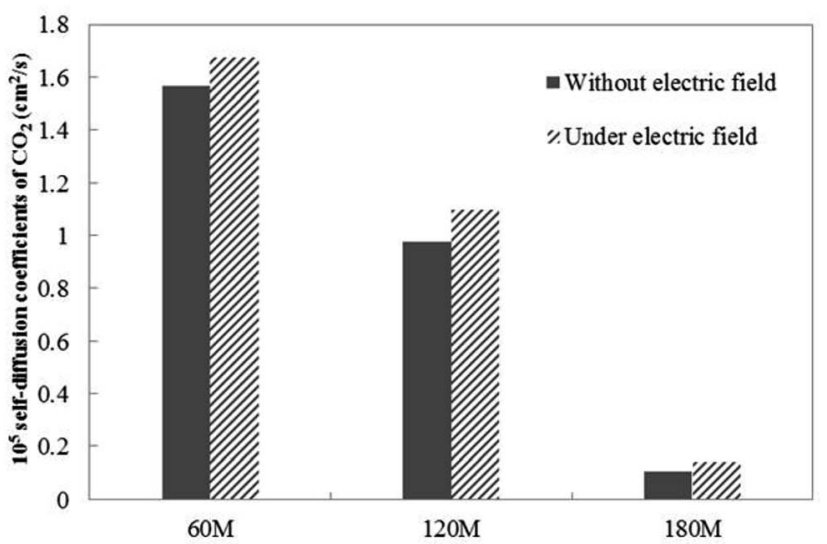

Fig. 4 The $\mathrm{CO}_{2}$ self-diffusion coefficient of systems in which different amounts of gas molecules are dissolved without an external electric field and under an external electric field.

Table 3 Geometrical parameters (height $H$, base radius $R_{\mathrm{b}}$, pressure $P$, volume $\mathrm{V}$ of bubbles

\begin{tabular}{llllrr}
\hline & & $H(\mathrm{~nm})$ & $R_{\mathrm{b}}(\mathrm{nm})$ & $P(\mathrm{~atm})$ & $V\left(\mathrm{~nm}^{3}\right)$ \\
\hline \multirow{2}{*}{ Without electric field } & $60 \mathrm{M}$ & 1.95 & 0.85 & 1242.38 & 6.10 \\
& $120 \mathrm{M}$ & 2.46 & 1.39 & 889.89 & 15.26 \\
& $180 \mathrm{M}$ & 2.97 & 1.38 & 797.58 & 22.60 \\
Under electric field & $60 \mathrm{M}$ & 1.95 & 0.82 & 1253.17 & 5.94 \\
& $120 \mathrm{M}$ & 2.46 & 1.35 & 901.00 & 14.83 \\
& $180 \mathrm{M}$ & 2.97 & 1.33 & 809.99 & 21.97 \\
\hline
\end{tabular}

where $v_{i, x}$ is the $x$ component of the velocity; $r_{i, z}$ is the $z$ coordinate; $m_{i}$ is the mass of an atom. The average of $V$ can be measured after the amplitude of the velocity profile has been fully developed.

Table 4 shows the viscosity of different systems. In Table 4, as the amount of dissolved gas increases, the viscosity also gradually increases. The viscosity value under the external electric field is lower than that without an external electric field. Normally, the diffusion coefficient of the solute in the solution is inversely related to the viscosity of the solution. The results in Table 4 are in good agreement with those in Fig. 4 . Viscosity can be seen as an expression of internal friction in solution. As the amount of gas dissolved increases, the total attraction of the hydrophobic substrate to the gas molecules becomes greater, while more gas molecules also increases the friction with the water molecules. Therefore, the increase of the amount of gas

Table 4 The viscosity in different systems ${ }^{a}$

\begin{tabular}{lcc}
\hline & $\begin{array}{l}\text { Without electric } \\
\text { field }\end{array}$ & $\begin{array}{l}\text { Under electric } \\
\text { field }\end{array}$ \\
\hline$V_{60 \mathrm{M}}(\mathrm{mPa} \mathrm{s})$ & 0.0340 & 0.0336 \\
$V_{120 \mathrm{M}}(\mathrm{mPa} \mathrm{s})$ & 0.0382 & 0.0378 \\
$V_{180 \mathrm{M}}(\mathrm{mPa} \mathrm{s})$ & 0.0456 & 0.0452 \\
$a$ “ $V_{60 \mathrm{M}}$ ", " $V_{120 \mathrm{M}}$ ”, " $V_{180 \mathrm{M}}$ " represent the viscosity of system with 60,120, \\
$180 \mathrm{CO}_{2}$ molecules, respectively.
\end{tabular}

dissolved leads to the increase of viscosity. When an external electric field is applied, some of the gas molecules in the bubble become more active, the gas molecules escaping from the bubble. They escape into the water phase, destroying the local hydrogen bond between the water molecules due to the movement of a small part of the gas, ${ }^{\mathbf{4 8 - 5 0}}$ resulting in the decrease of viscosity. Under the external electric field, the decrease of viscosity promotes a small portion of the gas escaping from the bubble to be more easily recoupled with the hydrophobic particles in the aqueous phase to form a new bubble, thereby enhancing the effect of separating the hydrophobic solid particles by air flotation.

\subsection{Hydrogen bond}

Hydrogen bonds between water molecules have a direct impact on the physical and chemical properties of solution system. ${ }^{\mathbf{5 1 , 5 2}}$ Hydrogen bonding is a special state of connection of water molecules, because water molecules keep moving irregularly in solution, the rotation of water molecules (i.e., the increase in hydrogen bonding angle) destroys the hydrogen bonds that have formed. ${ }^{53}$ In this work we employed the geometrical hydrogen bond criterion which is widely used in MD simulation. ${ }^{54}$ It requires the oxygen-oxygen distance to be less than $3.5 \AA$ and the $\mathrm{H}-\mathrm{O} \cdots \mathrm{O}$ angle to be less than $30^{\circ}$. In order to reduce the numerical origin, the results of this parameter are obtained from the average of five repeated simulations.

Fig. 5 shows that distribution of the hydrogen bond angle under an external electric field and without an external electric field. It can be seen that under no external electric field, the peak coordinates of the distribution of the hydrogen bond angle of systems with 60, 120, and 180 gas molecules are (9.5, 0.0554099), (9.5, 0.0545575) and (9.5, 0.0538870). The peak coordinates under an external electric field are (10.5, 0.0554477), (10.5, 0.0545804), (10.5, 0.0539568), respectively. Comparing the coordinates, it can be found that when there is an external electric field applied, the angle of the hydrogen bond generally becomes larger, and the rotation of the water molecule is more likely to break the original hydrogen bond. Thereby it causes a small part of the gas in the bubble easily escape to the water phase under the external electric field. When a part of the gas escapes from the bubble, the original hydrogen bond structure is destroyed, and the random movement of the water molecule is enhanced, which is consistent with the conclusion that the viscosity of the system is reduced under the external electric field.

Table 5 lists the proportion of water molecules containing 0 3 hydrogen bonds $\left(f_{0}(\%), f_{1}(\%), f_{2}(\%), f_{3}(\%)\right)$. As can be seen from Table 5, regardless of whether or not an external electric field is applied, the proportion of water molecules $\left(f_{0}\right)$ that does not form hydrogen bonds decreases as the amount of dissolved gas increases, and the proportion of water molecules that form hydrogen bonds $\left(f_{1}, f_{2}, f_{3}\right)$ increases. Compared with that under no external electric field, $f_{0}$ and $f_{1}$ increase under the external electric field, while $f_{2}$ and $f_{3}$ decrease, indicating that the external electric field reduces the number of water molecules containing two and three hydrogen bonds, the binding between 

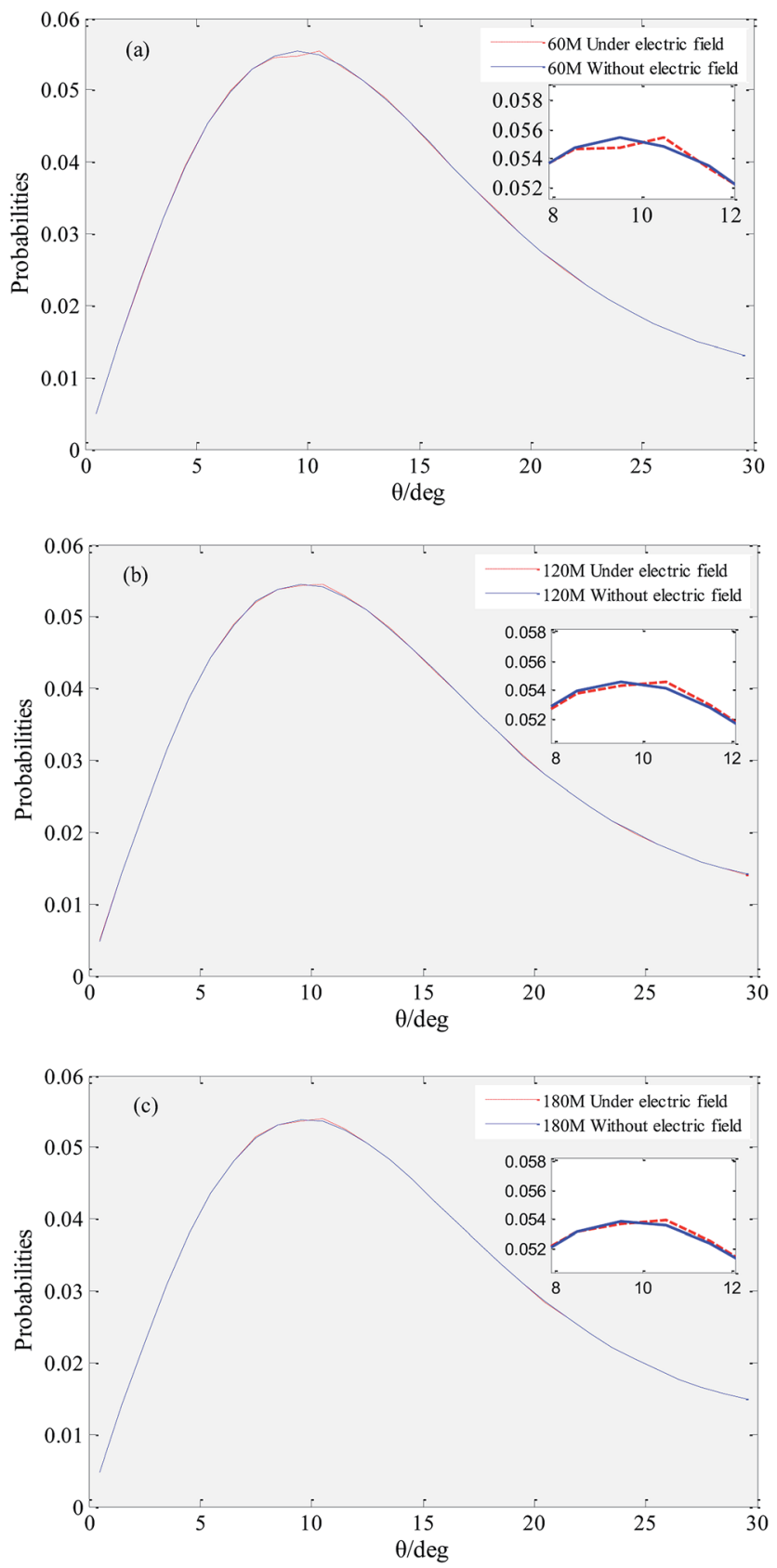

Fig. 5 Distribution of the hydrogen bond angle. (a), (b) and (c) show the hydrogen bond angular distribution of systems with 60,120 , and 180 gas molecules, respectively, under an external electric field (red dashed line) and without an external electric field (blue solid line).

water molecules decrease. It may because that the external electric field makes the bubble size become smaller, and some gas molecules escape to water phase cause the change of the proportion of hydrogen bonds between water molecules.

In addition, to clarity such changes are originated mostly by the presence of the $\mathrm{CO}_{2}$ molecules or by the electric field. The simulation that without $\mathrm{CO}_{2}$ molecules were also performed. The peak coordinate of hydrogen bond angle without an external electric field is $(9.5,0.0562010)$ and the peak coordinate of hydrogen bond angle under an external electric field is (9.5,
Table 5 The percentage of water molecules with $n$ hydrogen bonds $\left(f_{n}\right)$ in different systems

\begin{tabular}{|c|c|c|c|c|c|c|c|c|}
\hline & \multicolumn{4}{|c|}{ Without electric field } & \multicolumn{4}{|c|}{ Under electric field } \\
\hline & $0 \mathrm{M}$ & $60 \mathrm{M}$ & $120 \mathrm{M}$ & $180 \mathrm{M}$ & $0 \mathrm{M}$ & $60 \mathrm{M}$ & $120 \mathrm{M}$ & $180 \mathrm{M}$ \\
\hline$f_{0}(\%)$ & 39.65 & 39.89 & 39.71 & 39.59 & 39.50 & 39.90 & 39.73 & 39.60 \\
\hline$f_{1}(\%)$ & 36.12 & 36.07 & 36.08 & 36.10 & 36.16 & 36.09 & 36.11 & 36.12 \\
\hline$f_{2}(\%)$ & 19.61 & 19.50 & 19.58 & 19.65 & 19.67 & 19.49 & 19.56 & 19.64 \\
\hline$f_{3}(\%)$ & 4.62 & 4.54 & 4.63 & 4.66 & 4.67 & 4.52 & 4.60 & 4.64 \\
\hline
\end{tabular}

0.0562047 ) (figure or not given here). The results for $f_{n}$ were listed in Table $5(0 \mathrm{M})$, all the results show that the electric field enhanced the strength of the hydrogen bonds when there is no $\mathrm{CO}_{2}$ molecules existing in the system, which is consistent with Wei's work. ${ }^{55}$ But when there is $\mathrm{CO}_{2}$ molecules existing in the system, the results show that the electric field decreased the strength of the hydrogen bonds. Therefore, the results show that the changes are originated mostly by the electric field.

\section{Conclusions}

In this paper, molecular dynamics simulation was used to investigate the effect of external electric field on nanobubbles adsorbed at the surface of hydrophobic particles, and compared with results under no external electric field, some important conclusions are obtained:

(1) The results show that the size of bubbles is smaller when there is an external electric field applied. The smaller size of bubbles is beneficial to promoting the combination of bubbles and hydrophobic particles in the process of air flotation.

(2) As the amount of gas in the system increases, the diffusion coefficient of the gas molecules gradually decreases, which may be caused by the decrease of the internal pressure of the bubbles. The external electric field makes the gas molecules more active, and some of the gas molecules escape from the bubbles and escape to the water phase away from the hydrophobic substrate, so that the size of the bubbles under the external electric field become smaller.

(3) External electric field will reduce the viscosity of the system, when the bubble size becomes smaller, a small amount of gas escaped from the bubble will destroy the hydrogen bond structure in aqueous solution. Compared with that under no external electric field, the external electric field makes the angle of hydrogen bond between water molecules larger, the hydrogen bond is easier to be destroyed, and the proportion of water molecules with more hydrogen bonds decreases, and the hydrogen bond binding between water molecules weakens.

\section{Conflicts of interest}

There are no conflicts to declare.

\section{Acknowledgements}

This work was financially supported by the National Natural Science Foundation of China (Grant No. 51408525). 


\section{References}

1 J. L. Parker, P. M. Claesson and P. Attard, J. Phys. Chem., 1994, 98, 8468-8480.

2 L. Zhang, Y. Zhang and X. Zhang, Langmuir, 2006, 22, 81098113.

3 M. A. Hampton and A. V. Nguyen, Adv. Colloid Interface Sci., 2010, 154, 30-55.

4 S. Karpitschka, E. Dietrich and J. R. T. Seddon, Phys. Rev. Lett., 2012, 109, 066102.

5 V. S. J. Craig, Soft Matter, 2011, 7, 40-48.

6 C. Chan, L. Chen and M. Arora, Phys. Rev. Lett., 2015, 114, 114505.

7 T. Ito, H. Lhuissier and S. Wildeman, Phys. Fluids, 2014, 26, 032003.

8 V. S. Ajaev, Phys. Fluids, 2006, 18, 068101.

9 N. Kameda, N. Sogoshi and S. Nakabayashi, Surf. Sci., 2008, 602, 1579-1584.

10 J. R. T. Seddon, H. J. W. Zandvliet and D. Lohse, Phys. Rev. Lett., 2011, 107, 116101.

11 W. A. Ducker, Langmuir, 2009, 25, 8907-8910.

12 Y. Liu and X. Zhang, J. Chem. Phys., 2013, 138, 014706.

13 D. Tao, S. Yu and X. Zhou, Int. J. Coal Prep. Util., 2008, 28, 114.

14 Y. J. Tao, J. T. Liu and S. Yu, Sep. Sci. Technol., 2006, 41, 16. 15 M. A. Hampton and A. V. Nguyen, Miner. Eng., 2009, 22, 786792.

16 M. M. Fan, T. Daniel and R. Honaker, Int. J. Min. Sci. Technol., 2010, 20, 1-19.

17 Y. Han, C. Zhang and L. Wu, J. Cryst. Growth, 2018, 499, 6776.

18 N. Li, S. Tang and Y. Rao, Electrochim. Acta, 2018, 270, 330338.

19 L. Zhu and Y. Han, J. Mol. Liq., 2018, 271, 820.

20 J. M. Thiebaut, J. P. Vaubourg and G. Roussy, J. Phys. D: Appl. Phys., 1989, 22, 154.

21 M. Birinci, J. D. Miller and M. Sarıkaya, Miner. Eng., 2010, 23, 813-818.

22 M. Murugananthan, G. B. Raju and S. Prabhakar, Sep. Purif. Technol., 2004, 40, 69-75.

23 C. Li, A. Zhang and S. Wang, AIP Adv., 2018, 8, 015104.

24 J. H. Weijs, J. H. Snoeijer and D. Lohse, Phys. Rev. Lett., 2012, 108, 104501.

25 H. J. C. Berendsen, J. R. Grigera and T. P. Straatsma, J. Phys. Chem., 1987, 91, 6269.

26 P. Hong, G. R. Birkett and A. V. Nguyen, Langmuir, 2013, 29, 15266-15274.

27 L. Wang, X. Wang and L. Wang, Nanoscale, 2017, 9, 1078. 28 J. Li and F. Wang, J. Chem. Phys., 2017, 146, 054702.
29 J. G. Harris and K. H. Yung, J. Phys. Chem., 1995, 99, 12021. 30 X. Li, D. A. Ross and J. P. M. Trusler, J. Phys. Chem. B, 2013, 117, 5647.

31 H. Jiang, I. G. Economou and A. Z. Panagiotopoulos, Acc. Chem. Res., 2017, 50, 751.

32 W. L. Jorgensen, D. S. Maxwell and J. Tirado-Rives, J. Am. Chem. Soc., 1996, 118, 11225.

33 D. S. D. Van, E. Lindahl and B. Hess, J. Comput. Chem., 2005, 26, 1701-1718.

34 R. E. Iseleholder, W. Mitchell and A. E. Ismail, J. Chem. Phys., 2012, 137, 1133.

35 A. M. Saitta, F. Saija and P. V. Giaquinta, Phys. Rev. Lett., 2012, 108, 207801.

36 Q. G. Jiang, Z. M. Ao and D. W. Chu, J. Phys. Chem. C, 2012, 116, 19321.

37 P. Hong, M. A. Hampton and A. V. Nguyen, Langmuir, 2013, 29, 6123-6130.

38 Y. H. Lu, C. W. Yang and I. S. Hwang, Langmuir, 2012, 28, 12691-12695.

39 L. Zhang, X. Zhang and C. Fan, Langmuir, 2009, 25, 88608864.

40 X. H. Zhang, X. Zhang and J. Sun, Langmuir, 2006, 23, 17781783.

41 M. C. Zaghdoudi and M. Lallemand, Int. J. Therm. Sci., 2000, 39, 39-52.

42 P. Henritzi, A. Bormuth and F. Klameth, J. Chem. Phys., 2015, 143, 164502.

43 D. Y. Li, D. L. Jing and Y. L. Pan, Langmuir, 2014, 30, 6079.

44 M. P. Brenner and D. Lohse, Phys. Rev. Lett., 2008, 101, 214505.

45 B. Hess, J. Chem. Phys., 2002, 116, 209-217.

46 L. Zhao, T. Cheng and H. Sun, J. Chem. Phys., 2008, 129, 144501.

47 L. Zhu, Y. Han and C. Zhang, RSC Adv., 2017, 7, 4758347591.

48 V. S. J. Craig, B. W. Ninham and R. M. Pashley, Nature, 1993, 364, 317-319.

49 P. K. Weissenborn and R. J. Pugh, J. Colloid Interface Sci., 1996, 184, 550-563.

50 C. L. Henry, J. Phys. Chem. C, 2006, 111, 1015-1023.

51 W. J. Lee, J. G. Chang and S. P. Ju, Langmuir, 2010, 26, 1264012647.

52 Z. Pan, J. Chen and G. Lü, J. Chem. Phys., 2012, 136, 164313.

53 H. S. Lee and M. E. Tuckerman, J. Chem. Phys., 2006, 125, 154507.

54 X. Zhang, Q. Zhang and D. X. Zhao, Acta Phys.-Chim. Sin., 2011, 27, 2547-2552.

55 S. Wei, C. Zhong and H. Su-Yi, Mol. Simul., 2005, 31, 555559. 